\title{
Empathy in prairie voles: Is this the consolation prize?
}

\author{
Gregory E. Demas ${ }^{1}$ - Aaron M. Jasnow ${ }^{2}$
}

Published online: 21 July 2016

(C) Psychonomic Society, Inc. 2016

\begin{abstract}
Summary Although it is well known that humans and great apes are capable of engaging in consolation, an affiliative behavior directed toward distressed individuals, it has largely been assumed that this form of empathy was restricted to species possessing more complex cognitive functions. Recently, however, Burkett and colleagues (Science, 351, $375-378,2016$ ) have provided intriguing evidence that consolation behavior may be present in a socially monogamous rodent, the prairie vole. They also provide data to implicate the neuropeptide oxytocin in the regulation of this behavior, which suggests conserved neuroendocrine mechanisms between prairie voles and humans.
\end{abstract}

Keywords Classical conditioning $\cdot$ Fear conditioning $\cdot$ Social learning

In some ways, determining if nonhuman animals have the capacity to display empathy is like addressing the age-old theological question of "how many angels can dance on the head of a pin?" That is to say, it is a daunting, if not impossible, task. A recent paper by Burkett and colleagues (2016) in the journal Science, however, provides intriguing evidence that the prairie vole (Microtus ochrogaster), a socially mono gamous North American rodent, might be capable of displaying consolation behavior. Consolation is an affiliative

Gregory E. Demas

gdemas@indiana.edu

1 Department of Biology, Program in Neuroscience, and Center for the Integrative Study of Animal Behavior, Indiana University, Bloomington, IN 47405, USA

2 Department of Psychological Sciences, Kent State University, Kent, OH 44242, USA behavioral response aimed at providing physical and emotional comfort to a distressed individual; it is also a behavior that has been ascribed to a limited number of species to date, primarily but not exclusively to primates (de Waal \& van Roosmalen, 1979; Palagi, Stefania Dall'Olio, Demuru, \& Stanyon, 2014; Plotnik \& de Waal, 2014). Although consolation can be based on empathy, it is not a requirement; Burkett et al. are the first to empirically test if consolation behavior has an empathy-based mechanism in a rodent.

In this study, researchers demonstrated that prairie voles greatly increased partner-directed grooming, commonly called allogrooming, toward familiar animals, but not strangers, that had previously experienced a stressor unobserved by the test animal. Intriguingly, no such increase in allogrooming was directed toward animals that did not receive stress (i.e., control animals). Furthermore, when a closely related but nonmonogamous species of vole, the polygynous meadow vole (Microtus pennsylvanicus), was tested in a similar manner, no comparable changes in social behavior were observed. The authors suggest that this consolation behavior represents a form of "social buffering" that is specific to familiar conspecifics and is seen only in social species of rodents. To test the hypothesis that increased allogrooming was based on an empathy mechanism, the authors screened voles for commonly reported characteristics of human empathy; emotional contagion, state matching, familiarity bias, and self-other differentiation. One of the methods to examine emotional contagion was an auditory Pavlovian fear conditioning test in which freezing was measured while previously conditioned "demonstrators" and unconditioned (i.e., never shocked) "observers" were exposed to a single 30 -second conditioned stimulus (CS) tone. The observers showed increased freezing to the tone compared with freezing measured before the tone presentation, which was suggested to represent emotional contagion. 
Although there was a statistically significant effect, whether this response is biologically meaningful (e.g., increased chances for survival) remains to be seen. Freezing levels in the observers increased from 4 seconds (pretone) to 8 seconds during the tone presentation and are near baseline levels reported in many studies of fear conditioning, as is the $12 \%$ coordinated freezing between the observer and demonstrator. Although these results are clearly suggestive, future studies will need to determine if naïve (nonobserver) voles show a similar increase in freezing to tone, because presentation of a single novel tone can increase freezing in the absence of any conditioning.

To examine state matching, the authors measured corticosterone responses of observers and demonstrators under several social conditions. The nonstressed observer prairie voles appeared to "match" the response seen in stressed animals by showing comparable stress-induced increases in circulating levels of the adrenal glucocorticoid hormone corticosterone, but only when they were separated by a clear barrier. Increased corticosterone did not occur when animals were separated but not stressed. Contact with the demonstrator eliminated the increase in corticosterone, which was interpreted as state matching and suggested empathy toward the stressed cage mate. They further show familiarity bias and self-other differentiation by demonstrating increased allogrooming by observers toward a mate or sibling but not a stranger, and a specific increase in allogrooming by observers. To address the possible neurobiological mechanisms underlying this consolation response, the authors also performed an immunocytochemical analysis of FOS, the protein product of the c-fos gene that is commonly employed as a marker of neuronal activation, in response to exposure to a stressed or nonstressed animal. Last, they examined a potential role for the neuropeptide oxytocin (OT), which has been linked to emotional recognition, socioemotional engagement, and empathy. Specifically, if OT regulates, at least in part, consolation in voles, then blockade of the oxytocin receptor within the brain should inhibit this behavior. The results showed that the nonstressed observer displayed increased activity in the anterior cingulate cortex (ACC), and blockade of the OT receptors both throughout the brain and specifically within the ACC abolished this response. The results are compelling and consistent with the idea that OT plays a critical role in regulating empathic behavior in voles as in humans. These findings also suggest homology in the neurobiological mechanisms controlling consolation behavior across mammalian species. Furthermore, the importance of OT in consolation behavior in voles is perhaps supportive of recent speculation that the oxytocin system is conserved and responsible for the evolution of complex social behavior across vertebrate taxa, including the expression of human sociality (Carter, 2014).

Despite some of the noted limitations of this study, the findings from this impressive set of experiments demonstrate that prairie voles can detect when conspecifics have been previously stressed and can adjust some of their behaviors accordingly, responses consistent with consolation behavior seen in animals with more complex cognitive abilities (e.g., primates, canids, elephantids). What is clearly a more difficult task, however, is to know if these animals truly "understand" or "feel" what their stressed cage mates are experiencing, a key component of the empathic response, at least as it is understood in humans. The authors suggest that because the consolation test is an unconditioned response, it does not require cognitive capacity and therefore is a different form of empathy, functioning on instinctual motivations rather than cognitive empathy that is observed in humans. By redefining empathy based on unconditioned responses, the authors avoid the pitfalls inherent in trying to answer if observer voles truly "feel" what the "demonstrator" voles have experienced. Although these findings, or findings from other rodent studies, cannot easily address the cognitive aspects of empathy, they do support the idea that voles and other social animals are capable of what might be considered "affective empathy." In other words, although it is impossible to determine if the voles are consciously aware that their behavior is helpful to their stressed cage mates, it is clear that they are able to display behavioral responses that are specific to familiar individuals and that these responses can affect their own physiology. Although additional studies will surely be needed to address the question of empathic behavior in prairie voles or other nonhuman animals, and a definitive answer may forever be beyond our grasp, the careful and meticulous experimental approach taken by Burkett and colleagues might just be the "consolation prize."

\section{References}

Burkett, J. P., Andari, E., Johnson, Z. V., Curry, D. C., de Waal, F. B. M., \& Young, L. J. (2016). Oxytocin-dependent consolation behavior in rodents. Science, 351, 375-378.

Carter, C. S. (2014). Oxytocin pathways and the evolution of human behavior. Annual Review of Psychology, 65, 17-39.

de Waal, F. B. M., \& van Roosmalen, A. (1979). Reconciliation and consolation among chimpanzees. Behavioral Ecology and Sociobiology, 5, 55-66.

Palagi, E., Stefania Dall'Olio, S., Demuru, E., \& Stanyon, R. (2014). Exploring the evolutionary foundations of empathy: Consolation in monkeys. Evolution and Human Behavior, 35, 341-349.

Plotnik, J. M., \& de Waal, F. B. M. (2014). Asian elephants (Elephas maximus) reassure others in distress. Peer J, e278. 